\section{Diagnose und Behandlung der Frühstadien der Tabes.')}

\section{Von Prof. Friedr. Schultze in Bonn.}

Die Diagnose einer vollausgebildeten Tabes dorsalis ist geradezu kinderleicht; man muß nur nicht, wie das noch immer hier und da geschieht, das Hauptgewicht auf das Rombergsche Symptom legen. Denn diese Krankheitserscheinung tritt bekanntlich gewöhnlich erst in den vorgerückteren Stadien der Tabes ein, ist ferner auch bei anderen Krankheitszuständen zu finden und kann leicht fingiert werden, da sie schon seit lange den Laien bekannt geworden ist. Für die Diagnose der Tabes als solcher ist die Prüfung auf das Rombergsche Symptom fast geradezu unnötig geworden, wie man ohno Paradoxie sagen kann - sie spielt nur eine Nebenrolle. Die bekannten Hauptsymptome der Krankheit: lancierende Schmerzen (oft Rheumatismus genannt), Lichtstarre der Pupillen, Fehlen der Patellarreflexe, Hypästhesien, Hyperästhesien und Parästhesien sowie ausgebreitetere oder umschriebene Hypalgesien und Analgesien sind für die Feststellung einer beginnenden Tabes viel wichtiger und entscheidend.

Aber die meisten dieser Symptome führen den Kranken nicht zum Arzte. Erst wenn etwa die neuralgischen Schmerzen hartnäckiger werden, oder wenn gewisse weitere Symptome oft scheinbar ganz fernliegender Art den Kranken zu quälen beginnen, geht er zum Arzt und unter den heutigen Verhältnissen oft zuerst zu einem Spezialisten.

Von diesen Symptomen werden nach meiner Erfahrung am häufigsten noch immer die sogenannten gastrischen Krisen verkannt, die sich oft zeitlich zuerst bemerkbar machen und wohl auch das allererste Symptom der Krankheit darstellen können. Bekanntlich bestehen bei diesen Krisen rasch oder plötzlich auftretende Schmerzen in der Magengegend mit Erbrechen, zuerst von Speisen, dann von Schleim und Galle. Solch ein Anfall kann Stunden bis Wochen anhalten und bringt die Kranken sehr herunter. Daß solche Schmerzen mit Gallensteinkoliken, mit anderen Magenkrankheiten, mit Darmschmerzen, mit Pancreas- und Nierenkoliken verwechselt werden können, daß bei ihnen Appendicitis und epigastrische Hernien, selbst hysterische Zustände zugrunde liegen können, ist klar; ebenso daß sich die genannten Erkrankungen mit ihnen zu verbinden vermögen. Nötig ist aber, stets die genannten Reflexe zu untersuchen, zu denen auch noch die Achillessehnenreflexe gehören. Fehlen sie, oder sind sie nur einseitig vorhanden oder deutlich abgeschwächt, so ist bei Ausschlub anderweitiger Ursachen die Diagnose auf Tabes gegeben. Findet man dann noch daneben hypästhetische oder hyperästhetische horizontal gerichtete Querstreifen am Thorax oder am Abdomen oder umschriebene Analgesien an den Beinen oder am Rumpfe, so ist die Diagnose um so mehr gesichert, als diese Veränderungen der Sensibilität sich schon früh einzustellen pflegen. Bei genauer Anamnese wird man dann auch meistens noch das Bestehen von Blitzschmerzen, von Parästhesien, vielleicht auch von leichter eintretendem Ermüdungsgefühl, von beginnender geschlechtlicher Schwäche eruieren können.

Anstatt der vollausgebildeten Magenkrisen können aber auch nur Andeutungen von ihnen vorhanden sein, also einfache Cardialgien, plötzliche Uebelkeiten ohne nachweisbare weitere Ursache oder gar nur Anfälle von Pyrosis, bloße Neigung zu Magensäure, Kratzen im Halse, anfallsweise auftretender Speichelflub und ähnliche Symptome, wie sie jüngst Determann als rudimentäre Formen der Krisen beschrieben hat. Alle diese Krankheitserscheinungen sind selbstverständlich an sich viel zu vieldeutig und erst mehr retrospektiv nach Ausbildung deutlicher Magenkrisen und bei Vorhandensein anderer Zeichen von Tabes von Wert.

Gerade so wie Magenkrisen können auch zunächst die viel seltener auftretenden Darmkrisen den Kranken zum Arzte führen. Diese Störungen können in den verschiedenen Abschnitten des Darmrohres entstehen, auch im Mastdarm, so daß anfallsweise auftretende Schmerzen in diesem und im After mit oder ohne Stuhldrang anftreten (sogenannte Mastdarmkrisen), wobei natürlich nicht irgendwelche organische Veränderungen vorhanden sein dürfen, die diese Anfälle ausreichend erklären. Bei den eigentlichen Darmkrisen handelt es sich um plötzlich eintretende

1) Klinischer Vortrag.
Anfälle von Durchfällen ohne sonstige nachweisbare Ursache, meist ohne gleichzeitige Schmerzen.

Im Gebiete des Zirkulationsapparates können ebenfalls frühzeitig krisenähnliche Störungen entstehen, so daß dann die neuerlich sich immer mehr entwickelnden Herzspezialisten vielleicht zuerst in Anspruch genommen werden. Es zeigen sich in solchen Fällen Anfälle von Tachycardien, selbst von Herzschwäche, sodann von abnormen Empfindungen allerlei Art, die der Angina pectoris mehr oder weniger gleichen, oder endlich von einfachem, starkem Herzklopfen.

Im Gebiete des Respirationsapparates treten gelegentlich schwere Kehlkopfstörungen von vornherein in den Vordergrund, sodaß die Kranken zuerst zum Laryngologen gehen, der dann entweder eine Lähmung der Stimmbanderweiterer konstatieren kann oder bei sich im sensiblen Gebiete allein abspielenden anfallsweisen Reizzuständen keine Veränderung im Kehlkopfe vorfindet.

Wichtig ist ferner, daB manchmal zuerst trophische Störungen der Haut oder der Gelenke und Knochen die Kranken zum Arzte führen, und zwar diesmal hauptsächlich zum Dermatologen und zum Chirurgen. So kann besonders das sogenannte Mal perforant frühzeitig auftreten, oder schmerzlos aufgetretene starke Gelenkschwellungen und Gelenkveränderungen überhaupt sowie schmerzlos eingetretene Knochenbrüche können die Aufmerksamkeit selbst indolenter Kranken auf sich lenken. Hier und da kann auch einmal ein seltsamer Ausfall von Zähnen die Betroffenen zunächst zum Zahnarzte führen.

Von Bedeutung ist ferner die Tatsache, daß nicht selten Störungen im Bereiche der Blasenfunktionen und Erscheinungen von Impotenz zuerst sich bemerkbar machen und selbst jahrelang jedem anderen Zeichen von Tabes voraufgehen können, wie das z. B. Erb neuerdings wieder beschrieben hat.

Vielfach werden bekanntlich ferner die Sinnesorgane zuerst befallen, vor allem die Augen, deren Nervi optici oder deren Muskeln einer langsameren oder rascher eintretenden Lähmung verfallen können, wobei zu beachten ist, daß die Augenmuskellähmungen nur sehr schwach zu sein brauchen und bald vorübergehen können, während die Sehstörungen gewöhnlich mit eiserner Konsequenz zunehmen. Die Augenärzte sind es darum schon seit lange gewohnt, bei solchen Veränderungen zugleich auf anderweitige Störungen im Nervensystem zu untersuchen oder untersuchen zu lassen, während auf tabische Gehörsstörungen erst in den letzten Jahren häufjger hingewiesen wurde. Sie treten in Form von Ohrensausen und Gehörsabnahme auf, ohne sonstige objektive zur Erklärung der Erscheinungen ausreichende Ursachen.

Nimmt man nun noch dazu, dab auch cerebrale Erscheinungen verschiedener Art sich gleich von vornherein einstellen können, wie besonders Kopfweh oder Schwindel und sonstige als „neurasthen isch" geltende Symptome, vor allem größere Ermüdbarkeit beim Gehen, denkt man endlich daran, daß auch manchmal zuerst mehr der allgemeine Ernährungszustand in Form zunehmender Abmagerung aus unbekannter Ursache leiden kann, so hat man ein ungefähres Bild der Mannigfaltigkeit der Tabessymptome im Beginne der Erkrankung.

Um sie nun im Interesse des Kranken möglichst früh erkennen zu können, ist es erstens notwendig, jeden Kranken, ebenso wie man stets seinen Harn auf Eiweib und Zucker untersuchen soll, gleichgültig welche Beschwerden er hat, auch zugleich auf seine Pupillar- und Sehnenreflexe zu unter. suchen, und zweitens nach voraufgegangener Lues zu forschen, da diese nun einmal außerordentlich häufig in der Vorgeschichte der Tabes vorkommt, und da derjenige, der keine selbst erworbene oder angeerbte Syphilis gehabt hat, nur wenig Anrecht auf die echte Tabes hat. Ist Lues sicher dagewesen, so haben die kleinsten der erwähnten Einzelheiten eine große Bedeutung.

Glücklicherweise ist nun die Untersuchung auf die Pupillarund Sehnenreflexe, die vor allem vorgenommen werden muß, für gewöhnlich keine schwierige und zeitraubende.

Die Untersuchung auf die Lichtreflexe der Pupillen kann man sich, wie ich finde, dadurch besonders vereinfachen, daß man die Augenlider des Untersuchten mit den Fingern einfach zu- und aufklappt, wobei natürlich auch jedes Auge für sich untersucht werden muß. Manche Kranke zeigen sich allerdings dabei erregt und widerspenstig, sodaß man dann zu dem bekannten gewöhnlichen Verfahren schreiten kann, die Augen mit der vorgehaltenen Hand ausgiebig zeitweilig zu beschatten. 
Die Untersuchung auf die Patellarreflexe gestaltet sich sehr zweckmäßig, wenn man die Kranken auffordert, im Sitzen die Unterschenkel in einem stumpfen, nicht in einem rechten oder gar in einem spitzen Winkel, gegen die Oberschenkel zu bringen, während die ganze Fußsohle dem Boden aufruht. Dann beklopft man mit Hilfe des Jendrassikschen Handgriffes oder irgend einer Modifikation desselben die Patellarsehnen, bei möglichster Ablenkung des Kranken, prüft sowohl mit dem Auge als mit der aufgelegten Hand die Oberschenkelmuskeln desselben und sieht dabei auch auf die Sehnen selbst.

Die Achillessehnenreflexe lassen sich sehr gut so prüfen, daß man den zu Untersuchenden horizontal mit dem Bauche auf ein Bett oder ein Untersuchungssofa sich ausstrecken läßt und dann bei rechtwinklig gegen den Oberschenkel gestelltem Unterschenkel und bei leicht mit der einen Hand vorgenommener Dorsa]flexion des Fußes die Beklopfung der Achillessehnen vornimmt.

Wird auf diese Weise genau und eventuell öfters untersucht, so gewinnen schon geringere Abweichungen vom normalen, besonders aber stärkere Trägheit einer oder beider Pupillen bei jüngeren Leuten, einseitige Abschwächungen der Reflexe eine große Be. deutung, wobei selbstverständlich auch an andere Ursachen für dieses pathologische Verhalten gedacht werden muB. Im besonderen sei daran erinnert, dab bei Potatoren vor allem die Achillessehnenreflexe fehlen können und daß bei sehr fetten Leuten mit kurzen Patellarsehnen, wie schon $\mathrm{C}$. Westphal fand, die zugehörigen Reflexe sehr schwach oder selbst fehlend sein können.

Hat man auf diese Weise unzweifelhafte Abweichungen vom Normalen festgestellt, so ist vor allem auf die erwähnten $\mathrm{Hyp}$ ästhesien und Hypalgesien am Rumpf und an den Extremitäten zu untersuchen, sowie auch besonders auf Hyperästhesien, vorzugsweise gegenüber Kälte, zu achten. Finden sich auch diese und sind dann gar noch die so häufigen blitzartigen Schmerzen, der sogenannte "Rheumatismus", zu konstatieren, so ist die Diagnose auf Tabes gesichert und die Bedeutung der aufgezählten selteneren Frühsymptome festgestellt. Freilich ist dabei selbstverständlich zu beachten, daß auch neben beginnender Tabes irgend welche anderen Leiden zugleich bestehen können, die etwa Cardialgien, Krisen etc. hervorzurufen vermögen. So sah ich in einem Falle gastrische Tabeskrisen und Gallensteinkoliken nebeneinander. In neuester Zeit legt man auf die Cytodiagnostik der durch die Lumbalpunktion entnommenen Spinalflüssigkeit Wert, eine Untersuchungsmethode, die noch weiterer Prüfung bedarf und sich vorläufig für den allgemeinen Praktiker noch nicht eignet.

Wichtig ist aber, sich gegenwärtig zu halten, daß auch das Erhaltensein von Pupillar- und Patellarreflexen noch nichts gegen eine beginnende Tabes beweist. Man braucht auf das Fehlen dieser Reflexe nicht erst zu warten, wenn man bei einem früher syphilitisch Gewesenen lancinierende Schmerzen oder eines jener erwähnten Symptome besonders auf dem Gebiete der Sensibilität vorfindet. Umgekehrt besteht auch nicht selten etwa nur eine einseitige Lichtstarre der Pupillen, ohne andere Symptome, sodaß es dann freilich fraglich bleibt, ob es sich um eine im ersten Beginne stehende oder stationär gewordene Tabes oder progressive Paralyse handelt, ob also die gleichen anatomischen Veränderungen zugrunde liegen wie bei der gewöhnlichen Tabes oder nicht. Immerhin tut man in derartigen zweifelhaften Fällen gut, therapeutisch so zu handeln, als wenn schon ausgesprochene Tabes vorhanden wäre.

Denn ist die Diagnose auch nur wahrscheinlich gemacht, dann muß sogleich die zweckmäbige Therapie eingeleitet werden. Man wird dem Kranken, wenn er früher an Lues oder auch nur an zweifelhafter Syphilis gelitten hat, schonend eröffnen müssen, daß nicht etwa bloß eine "Neurasthenie" oder ein örtliches funktionelles Nervenleiden vorliege, sondern daß eine ernste Erkrankung entstehen könne, wenn er nicht konsequent das Richtige tue und geschehen lasse.

Dieses Richtige besteht vor allem in der Abhaltung von Schädlichkeiten jeder Art und in der Einhaltung einer nach jeder Richtung hin soliden, verständigen und mäßigen Lebensweise. Da glücklicherweise nicht jeder Syphilitische Tabes bekommt, eine Tatsache, die man, nebenbei bemerkt, gegen den $\mathrm{Zu}$ sammenhang von Tabes und Lues geltend gemacht hat, als ob etwa jeder Diphtheriekranke eine Diphtherielähmung oder jeder Kranke mit akutem Gelenkrheumatismus eine Endocarditis oder
Pericarditis bekäme, so müssen in jedem Einzelfalle noch weitere mitwirkende Veranlassungen da sein, die das Nervensystem und einzelne Teile desselben besonders empfänglich machen. So ist gewiß anzunehmen, daß Leute, die ihr Nervensystem überanstrengen, leichter Tabes bekommen als andere, ebenso wie es wahrscheinlich ist, daß jemand, der ein chronisches sonstiges Magenleiden hat oder einen schwächer von Hanse aus beschaffenen Magen, leichter gastrische Krisen bekommen kann als andere. Also Enthaltung von allen seelischen, geistigen und körperlichen Anstrengungen oder gar Ueberanstrengungen, Sorge für guten und langen Schlaf, für lange Ruhepausen, für Enthaltsamkeit oder weitestgehende Beschränkung in bezug auf Alkohol, Tabak und besonders auch auf Geschlechtsgenüsse! Und zwar muß ein solches Regime für die ganze Lebenszeit durchgeführt werden.

Sodann steht an der Spitze der Behandlung eine zweckmäßige, lange und mit Pausen fortgesetzte antisyphilitische Behandlung. Quecksilberkuren können mit Jodkuren in der Art abwechseln, daß zuerst eine Quecksilberkur vorausgeschickt wird, später alle paar Monate etwa ein Monat lang die bekannte gewöhnliche Dosis von Jodkalium oder Jodnatrium dargereicht wird. Nach $1 / 2-1$ Jahr kann dann von neuem eine Quecksilberkur folgen. Gleich vielen Anderen bevorzuge ich die alte Einreibekur, die sich allerdings nicht mit Herumgehen während des gröbten Teiles des Tages verträgt, sondern als Einatmungskur angesehen werden muß. Ich folge dabei speziell den bekannten Vorschriften von Neisser. Gegenüber der dauernd drohenden Gefahr von Verschlimmerungen muß eine derartige Behandlungsmethode selbstverständlich in individualisierender Weise jahrelang fortgesetzt werden, und mindestens dann sofort wieder aufgenommen werden, wenn sich neue Krankheitssynptome den alten hinzugesellen und alte, besonders Schmerzen, zu lästig werden.

Innere spezifische Mittel gegen die Krankheit gibt es nicht, ebensowenig wie äußere, mögen sie in Elektrizität, Bädern oder gar in Massage oder Suspension bestehen. Aber man wird sowohl sie als auch besonders das Argentum nitricum immer wieder von neuem versuchen können. Dagegen müssen selbstverständlich gegen die verschiedenen Einzelsymptome quälender Art gewisse Mittel gebraucht werden, ebenso wie die Besserung des Allgemeinzustandes durch geeignete Maßnahmen angestrebt werden muß.

In letzterer Beziehung erfreuen sich mit Recht die kohlensauren Bäder und kohlensauren Salzbäder besonderer Beliebtheit, besonders wenn sie nicht zu Hause gebraucht werden, sondern wenn der Kranke sie in den entsprechenden Badeorten selbst gebraucht. Denn fern von seiner gewohnten häuslichen Tätigkeit kann der Kranke draußen zugleich der Ruhe pflegen und vielfach leichter nur seiner Gesundheit leben als daheim. Daß aber auch in anderen Badeorten, etwa solchen mit Schwefel- oder Jodbädern, oder bei einem Aufenthalt an der See oder in einem passenden Luftkurorte das Gleiche erzielt werden kann, ist selbstverständlich. Zweckmäßigerweise können auch die Hg-Kuren zugleich mit einem derartigen Aufenthalte verbunden werden, wenn auch für diesen Zweck der Aufenthalt in einem geeigneten Sanatorium noch für förderlicher angesehen werden mub.

Bei der Bäderbehandlung sei aber auch hier die alte Warnung wiederholt, Tabische mit lancinierenden Schmerzen wegen ihres vermeintlichen "Rheumatismus" nicht mit heißen Prozeduren zu beglücken, ebensowenig wie kalte Güsse und eine energische Kaltwasserbehandlung am Platze sind. Tabische müssen eben von Anfang an schonend behandelt werden! Gegen die Schmerzen ist der Kampf oft schwierig. Wie anderen, so hat sich auch mir in den letzten Jahren das Py ramidon von den innerlichen Mitteln am meisten wirksam erwiesen; auf jeden Fall mub man mit dem Morphium so zurückhaltend wie möglich sein. Blutige Nervendehnungen in besonderen Notfällen werden wohl jetzt nicht mehr vorgenommen, unblutige, Suspensionen mäßiger Art, Massage, Elektrizität mögen versucht werden, schon um den Kranken nicht verzweifeln zu lassen oder ihn nicht dem Morphinismus zuzuführen.

Gegen frühzeitige Blasen- und Geschlechtsschwäche ist die Anwendung des elektrischen Stromes und die innerliche oder subcutane Applikation von Strychnin und Nux vomica am Platze.

Gegen die Magenkrisen ist oft besonders schwer aufzukommen. Selbstverständlich ist in den Zwischenpausen zwischen den einzelnen Anfällen mit allen unseren neueren Hilfsmitteln die etwa bestehende chronische Magenerkrankung zu bekämpfen, wobei hervorzuheben ist, dab keinesfalls stets eine Hyperacidität 
auch während der Anfälle selbst sich nachweisen läßt. In den Anfällen selbst läßt sich die Anwendung von Morphiumeinspritzungen nicht umgehen. Man wird aber daneben versuchen, durch Einläufe mit Codein, Dionin und den verschiedenen Schlafmitteln die oft scheußlichen Zustände zu bessern. Die innerliche Darreichung der genannten Mittel, zu denen auch noch die Belladonna zu rechnen ist, wird ja oft genug durch das leicht und oft eintretende Erbrechen gehindert.

Die A taxie gehört gewöhnlich nicht zu den Frühsymptomen der Tabes, von deren Behandlung hier wesentlich die Rede ist. Ist sie aber auch nur in ihren Anfängen da, so gilt es besonders streng auf die erwähnten allgemeinen Maßnahmen zu dringen und dann frühzeitig in vorsichtiger Weise mit den hauptsächlich von Frenkel ausgebildeten Bewegungsübungen zu beginnen. Auch ohne kompliziertere Verrichtungen lassen sich diese in der Weise einrichten, daß der Kranke, auf einer Chaiselongue liegend, bis zu eben eintretender Ermüdung, lieber also häufiger, verschiedene Zielbewegungen macht, sodab er gegen Punkte auf einer am Ende der Chaiselongue befindlichen Tafel stößt oder Kreisen und anderen Figuren, die auf ihr angebracht sind, mit dem Fube folgt etc. Ebenso kann er einfache Gehübungen längs einer vorgezeichneten Linie machen, rückwärts gehen etc.

Verfährt man auf diese Weise, dann kann man viel länger als früher die Kranken vor dem zweiten atactischen Stadium ihrer Erkrankung bewahren, mit dessen Behandlung man früher erst begann. Man kann wohl auch den Eintritt dieses Stadiums überhaupt verhüten und ist somit mit Hilfe unserer heutigen verfeinerten Diagnostik und mit Hilfe unserer genaueren ätiologischen Kenntnisse über die Krankheit derselben gegenüber lange nicht mehr so ohnmächtig wie früher. 